\title{
REQUIREMENTS FOR AERIAL IMAGES QUALITY, OBTAINED FOR MAPPING PURPOSES
}

\author{
Irina Anikeeva $^{1 *}$, Aleksandr Chibunichev $^{2}$ \\ ${ }^{1}$ JSC ”Roscartography”, Moscow, Russia - ia.anikeeva@ roscartography.ru \\ ${ }^{2}$ Moscow State University of Geodesy and Cartography, Moscow, Russia - agchib@miigaik.ru
}

KEY WORDS: Aerial Image, Aerial Image Quality, Blurring, Random Image Noise, Haze, Color Balance, Loss of Information in Shadows and Illumination

\begin{abstract}
:
The quality of cartographic products, obtained on base of aerial images, depends on their quality. The image's quality can be defined in terms of measurement characteristics and in it's visual properties. The object of the paper is an aerial image's quality, concerning its visual perception. The process of topographic aerial images quality assessing is currently not regulated, therefore, this problem becomes particularly relevant today. The article is devoted to development the quality criteria system for aerial images, obtained for mapping purposes, and definition the requirements for them. The analysis of factors affecting the quality of aerial images is carried out, they include natural conditions, technical and technological parameters of aerial surveying and images post-processing. The main imperfections of image quality, appearing as a result of these factors influence are determined - blurring, high level of random image noise, haze, color imbalance, loss of information in shadows and illumination. Methods of identifying these imperfections and assessing their influence on aerial images quality are shown.
\end{abstract}

\section{INTRODUCTION}

Currently, a wide range of large-scale cartographic products (maps and plans, orthophotomosaics, 3D terrain models, textured with photorealistic images, stereoscopic terrain models) are created by aerial imagery. That's why aerial images, obtained for mapping purposes, become a subject of high requirements in terms of measurement properties. However, the quality of resulting products depends not only on measuring properties of images, it is equally influenced by their visual characteristic. It is obvious that even with high-precision geodetic reference of an image, which has a low visual quality (for example, unsharp), and, as a result, poor interpretation properties, it is not possible to perform the accurate measurements on it either in manual or automatic modes.

The quality of analog aerial images was regulated in terms of photogrammetric and photographic (pictorial) properties. In terms of photogrammetric characteristics (image's inclination angles, overlap, etc.), these requirements have not lost their relevance for images, obtained by modern digital airborne cameras. But, in terms of pictorial properties, requirements for analog aerial images are not applicable for digital aerial photographs.

The theme of mapping image quality assessment raised in sufficient number of scientific works. Most often they concern the estimation of a single criteria - spatial resolution (Lim et al, 2018; Meißner et al, 2018; Orych, A., 2015). However, spatial resolution does not always allow to give a complete description of the image's quality. For this reason, the problem of development the unified technique, based on numeric criteria system, for topographic aerial images quality assessing becomes particularly relevant today.
The aim of the study is development of the numeric quality criteria system for aerial images, obtained for mapping purposes, and define the requirements for them. On this criteria system can base the unified technique for images quality assessing in terms pictorial properties.

\section{METHODOLOGY}

To develop the quality criteria system for aerial images and to define the requirements for them it is necessary the following tasks to be solved: the analysis of factors, affecting the aerial images quality; determination of images quality imperfections, that occur due to the influence of these factors; determination the ways to identify these imperfections and assess the extent of their influence on images quality.

\subsection{Factors affecting the aerial images quality}

The aerial image quality is determined by the following main factors (Kuchko, 1974; Srivastava, 2014):

1. natural surveying conditions, the main of which are the illumination and the atmosphere condition,

2. technical surveying conditions - carrier evolutions during aerial surveying, technical features of airborne camera (presence/absence of forward motion compensation (FMC), shutter type, etc.),

3. technical surveying parameters, such as exposure time, sensor sensitivity settings, etc.,

4. technological parameters of images post-processing radiometric correction, histogram transformation, etc.

The influence of these factors, if they are closely related, can result in decreasing of the image quality. Below are the main

\footnotetext{
${ }^{*}$ Corresponding author
} 
imperfections in aerial images, caused due to the influence of the listed factors.

\subsubsection{Image's blurring (including motion blurring)}

Why: linear and angular carrier motions during aerial surveying. Appearance: edges on image are not clear contrast lines, but blurred transition bands.

Sharpness of an image is defined as the overall blurring of edges and the blurring of details is defined as it's clarity ${ }^{2}$. The sharpness and clarity of an image decreases with increasing the light sensitivity of the matrix. This fact especially should be kept in mind for large-scale topographic aerial surveys.

The problem is particularly relevant because of the increasingly active use in practice of aerial surveying the imaging systems based on unmanned aerial vehicles (UAV). UAVs have a small mass, that's why it's difficult for them to maintain their position stability when performing aerial surveys. In addition, the most widely used UAV survey systems are equipped with nontopographic cameras, in which there is no FMC. UAV payload limitations also prevent the installation of onboard gyrostabilizing platforms.

Figure 1 shows two fragments of aerial images, obtained by the UAV. Despite the fact that the images were taken under the same conditions (during one flight at the same height), they have visually different sharpness.

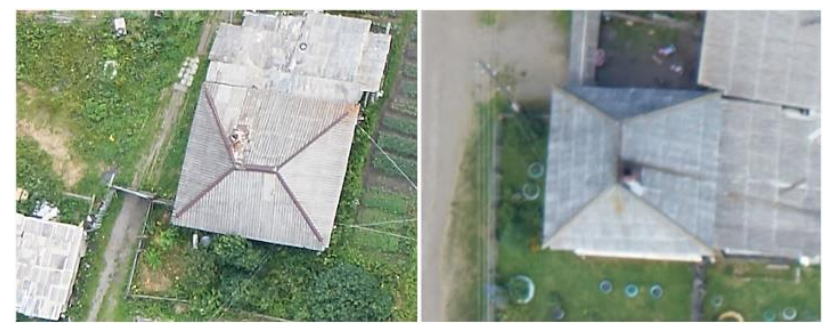

Figure 1. Fragments of aerial images with different sharpness (clarity): the sharpness of the right image is noticeably lower than that of the left image

Visually, the blurriness of an image can be estimated by sharp edges of objects by visible pixelation at high magnification. The edge transition is a site of an image (a part of a row/column), containing the sharp change in pixels tone (values) from light to dark on a relatively small pixel interval (about several pixels) (Anikeeva, Kadnichanskiy, 2017). For images of visually high sharpness (clarity), the length of an edge transition does not exceed 5 pixels. If the edge transition is 5 or more pixels long, the image looks blurry, that reduces its interpretation and information properties, it also results in errors in recognition and measuring both in manual and automatic modes (e.g. less accuracy of the correlator). Figure 2 shows an example of edge transitions for sharp blurry image.
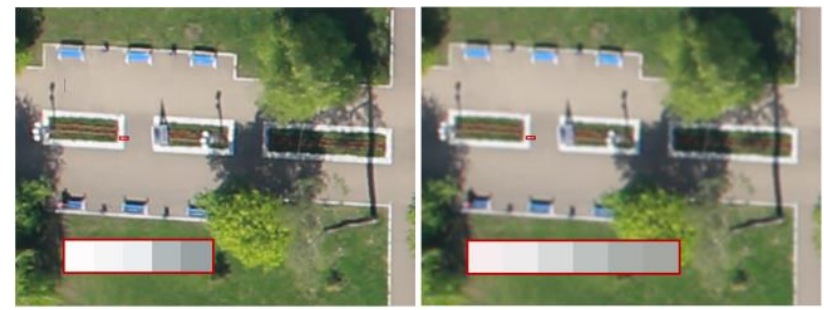

\footnotetext{
2 Geodetic, Cartographic Instructions, Norms And Rules (GCINR)-02-121-79 Guide For Aerial Images Interpretation When Surveying And Updating Plans At Scales Of 1:2000 And 1:5000
}

Figure 2. An example of edge transitions in a sharp image (left) and in a blurry image (right)

If an image has areas of illumination, they also have edge transitions, shown in Figure 3. However, such edge transitions are related to artifacts and cannot be used to assess the image's sharpness.

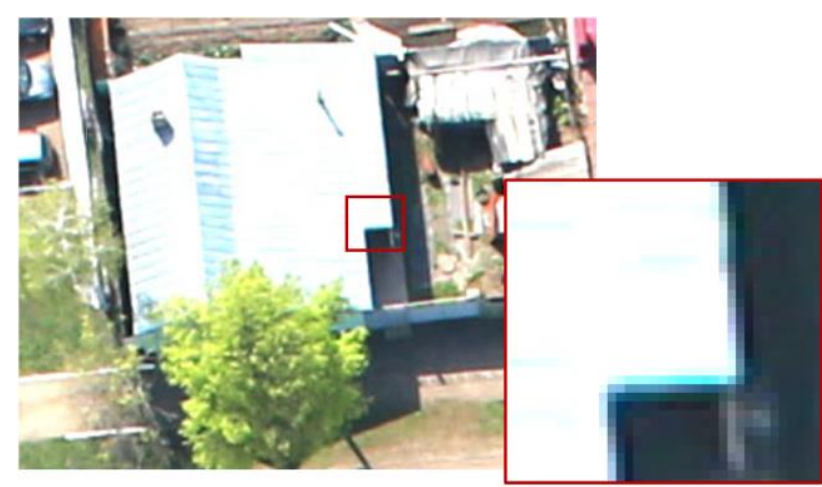

Figure 3. An example of edge transitions in the area of illumination, they should not be used them for assessing the images sharpness

Motion blur is a special case of image blurring. It is characterized by blurring in one direction, usually in the direction of camera's moving.

Why: camera motion during the exposure.

Appearance: double vision of edges, geometry distortion (for example, neighbour equal compact objects, such as cars, curbstones, road markings, etc., that have the same size and shape, are displayed in the image as objects of different size and area, depending on their orientation relative to the axis of the flightline), an example shown in Figure 4.

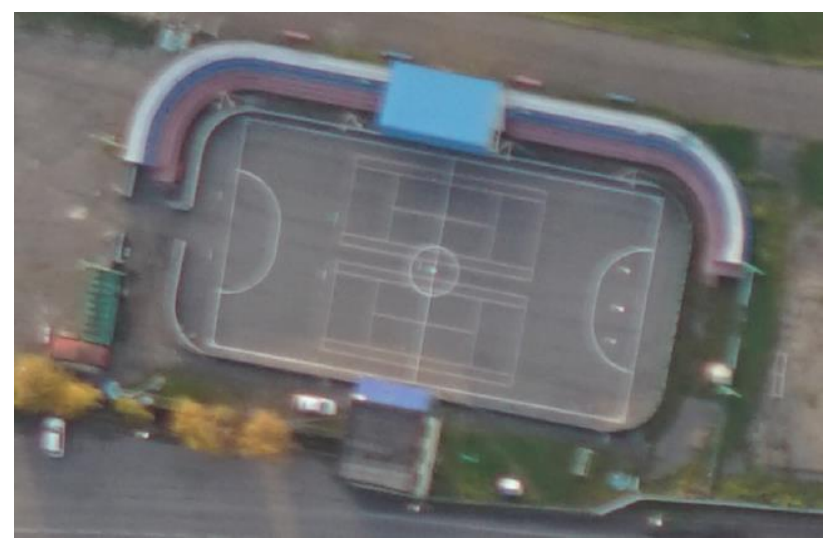

Figure 4. An example of double vision of edges and objects geometry distortion caused by motion blur

\footnotetext{
Methods of blur assessment: $\square \quad$ visually based on sharp edges at high magnification, numerically with formula for the normalized length of the edge transition $l_{\text {norm }}$ :
} 


$$
l_{\text {norm }}=\frac{3}{L}
$$

where $\quad L=$ edge transition length in pixels

Sharpness index $l_{\text {norm }}$ is a normalized value, that varies from 0 to 1 . The closer $l_{\text {norm }}$ to the value 1 , the better an image's sharpness and, accordingly, the closer it is to the value 0 , the blurry an image looks.

Images with a high contour load are selected to assess the sharpness index, e.g. built-up area, industrial zones, in which the sharp edges are present. Edge transitions oriented along rows or columns of an image have the highest gradient. In addition, the sharpness of an image in these directions may also be different, e.g., in the case of motion blur, when the sharpness along the flightline is lower than the sharpness in perpendicular direction. Therefore, the sharpness index should be calculated in two directions of an image - along rows $l x_{n o r m}$ and along columns lynorm.

If the difference between $l x_{n o r m}$ and $l y_{n o r m}$ is not significant (in the second decimal place), there is a general blurring in the image. If the difference between $l x_{n o r m}$ and $l y_{\text {norm }}$ in the first decimal place, there is a motion blur, directed along the axis where the sharpness index is minimum.

For visually sharp images $l x_{\text {norm }}$ and $l y_{\text {norm }}$ exceed the value of 0,5 .

Numerical sharpness assessment can be performed both in manual and automatic mode. In case of manual sharpness assessment edge transitions are being selected visually at a high magnification.

Image blur also decreases it's spatial resolution. The rated image spatial resolution is determined by the pixel size projection on the underlying surface. The actual spatial resolution is determined by the smallest object's linear dimensions, visible in the image, defined both in pixels and in meters. It depends not only on physical pixel size and the surveying height to camera's focal length ratio, but also on the image's quality - blur, noise, etc.

Spatial resolution is conventionally assessed by the specialized test-objects, such as 1951 USAF resolution test chart, Siemens star, ISO 15775 chart and others. Such tests are carried out in the laboratory or on a special polygon.

The task of images quality assessment always arises during production performance of aerial surveying for mapping purposes. In this case the application of test-objects is impractical and nine times of ten is impossible because it is necessary to assess the quality of each image, that accepted for further photogrammetric processing. For these purposes methods based on modulation transfer function (MTF) analysis are used (Lim et al, 2018; Takahashi et al., 2020). One of these methods described in (Anikeeva, Kadnichanskiy, 2017). The algorithm consists in automatic search the edge transitions with maximum gradient in the image and their MTF analysis. The advantage of proposed method is that the spatial resolution and sharpness assessment by formula (1) are performed at the same time by the same edge transitions.

\subsubsection{Haze}

Why: the influence of atmospheric transparency.

Appearance: reducing the brightness interval of landscapes and the possibility of clear separate reproduction the topographic objects on aerial images (mainly in shadows). An example of atmospheric haze influence on interpretation quality of an aerial image is show in Figure 5.

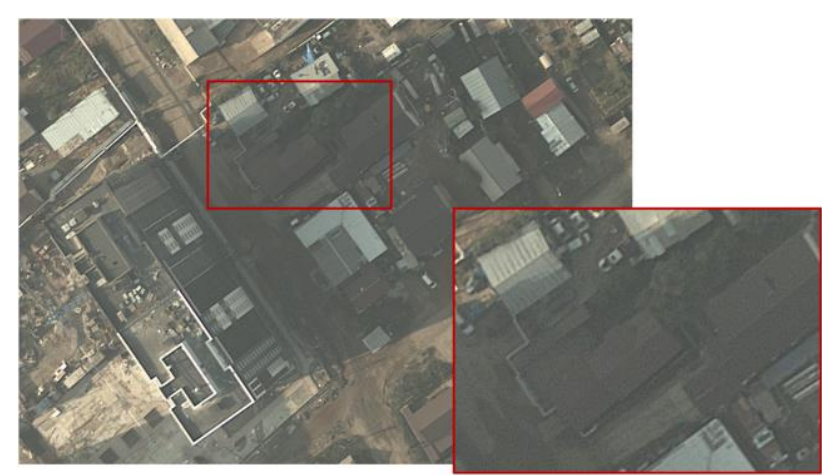

Figure 5. An example of atmospheric haze influence on interpretation quality of an aerial image

To avoid the haze appearance on images the aerial surveying should be performed when the haze is not visually perceived and the horizontal meteorological visibility range is not less than 10 $\mathrm{km} .{ }^{3}$

Methods of haze assessment:

$\square \quad$ visually as general bright background in image and the decrease of contrast,

$\square \quad$ numerically with the sequence of zero histogram values in the area of low pixel brightness, an example of haze detection by histogram is shown in Figure 6 .

Haze detection by histogram can be performed both visually and automatically by implementing the simplest algorithms in any software environment, that allows to build an image histogram and perform math actions with it, e.g. Mathcad, MATLAB, Python, etc.

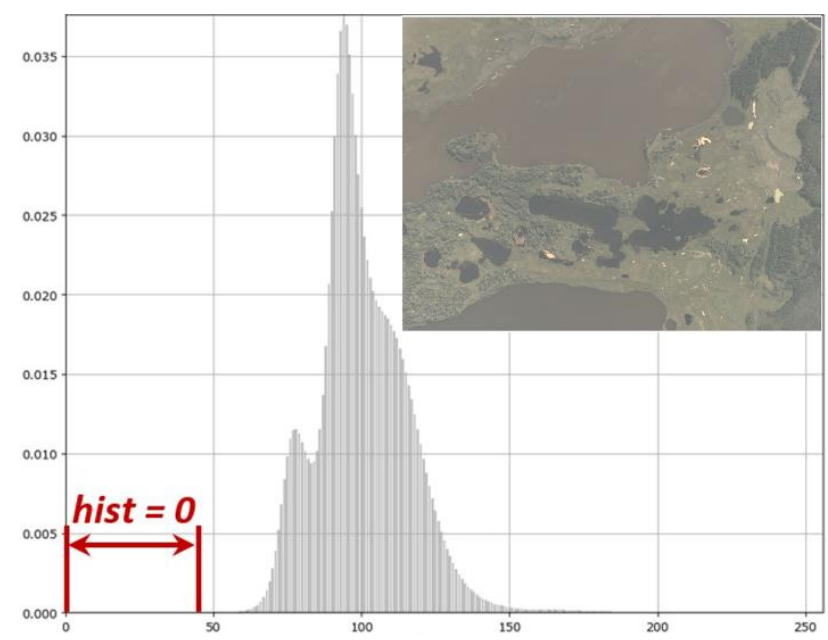

Figure 6. An example of a histogram for aerial image with haze with the distinctive zero values sequence in the area of low pixel brightness

\subsubsection{Loss of information in shadows and illumination}

Why: wrong exposure selection; limitations of the photosensitive matrix's dynamic range; incorrect images postprocessing. Appearance: polygons of pixels with maximum brightness, indicating the loss of information in illumination; deep shadows on aerial images that prevent identification of objects caught in them.

\footnotetext{
${ }^{3}$ GCINR-02-121-79 Guide For Aerial Images Interpretation When Surveying And Updating Plans At Scales Of 1:2000 And 1:5000
} 
An example of information loss in shadows and illumination on aerial images is shown in Figure 7.
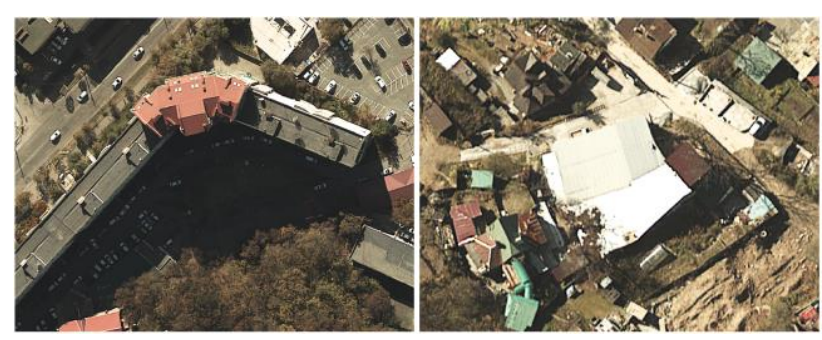

Figure 7. Loss of information in shadows (left) and illumination (right) on aerial images

The presence of large areas of illumination on aerial images is unacceptable. Single cases that unavoidably occur in clear sunny weather as a result of direct sunlight reflection from smooth surfaces (roofs of buildings, water surfaces) are allowed as an exception. Such areas should occupy a small area, be local and not prevent the object's interpretation. Objects on images should be displayed without structural and textural property losses in illumination. At the same time images should have a good details elaboration in shadows, i.e. features that fall into the shadow of high-rise objects should be definitely displayed and well interpreted, as shown in Figure 8.

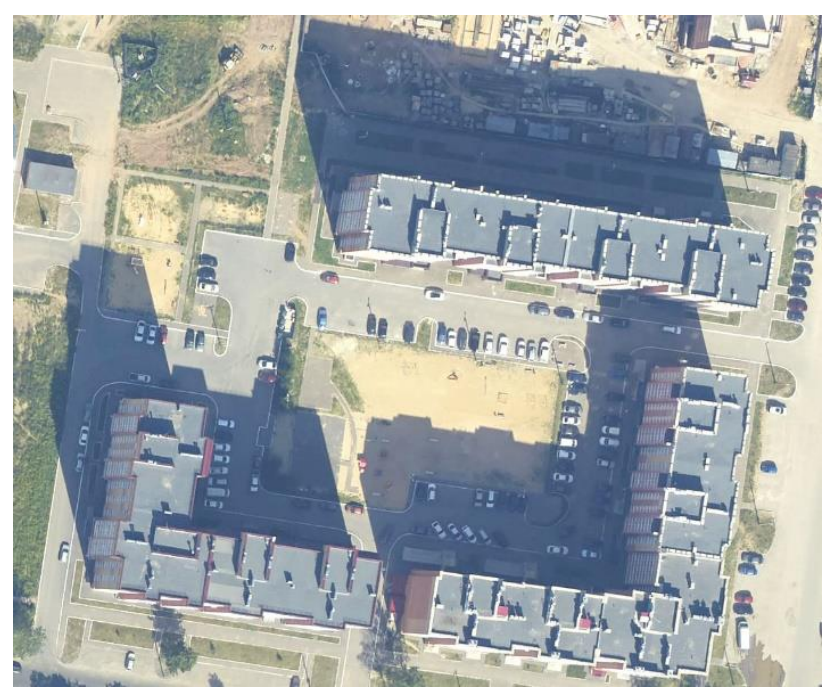

Figure 8. An example of good details elaboration in shadows in aerial image

Methods of information loss assessment in shadows and illumination:

$\square \quad$ visually on image, maximum possible pixel value's outliers.

With sufficient radiometric resolution (Chandra, Gosh, 2008) the histogram has an outlier in minimum or maximum pixel value (or in both at once in case of information loss in shadows and illumination at the same time). Figure 9 shows an example of an image with haze and information loss in shadows and illumination.

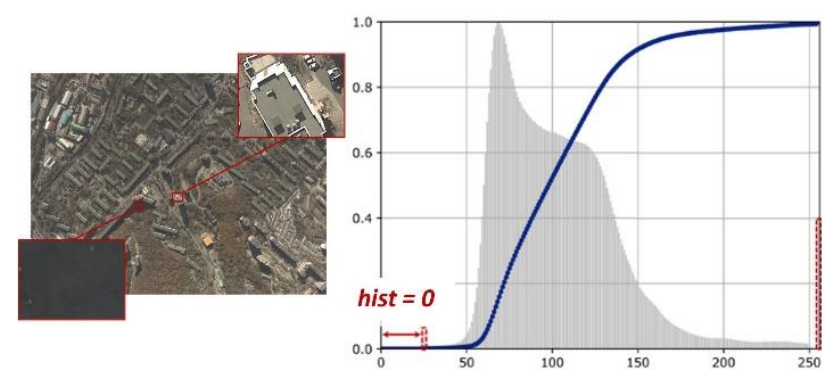

Figure 9. An example of an image with haze and information loss in shadows and illumination, detected by histogram

Numerically, information loss in shadows and illumination can be estimated as percentage of total number of pixels in the image, i.e. the percentage of information loss in shadows is the ratio of number of pixels with minimum value (if there is a histogram outlier at this pixel value (see Figure 9) to total number of pixels in the image; and the percentage of information loss in illumination is the ratio of number of pixels with maximum possible value, provided by image's radiometric resolution $(255$ for radiometric resolution 8 bits/pixel) to total number of pixels in the image.

For example, the image's size in Figure 9 is $9000 \times 6732$ pixels, so the total number of pixels is 60588000 . The number of pixels with minimum value is 32657 , and the number of pixels with value 255 is 374676 . The information loss in shadows for this image is $\approx 0,05 \%$, and the information loss in illumination is $\approx 0,62 \%$. Figure 9 shows, that even a very small percentage of information loss leads to significant decrease in image's interpretation quality.

In the case of radiometric resolution lack, the histogram has the form of a truncated figure in minimum or maximum pixel's brightness value (or both at once), the example of such image and it's histogram is shown in Figure 10.

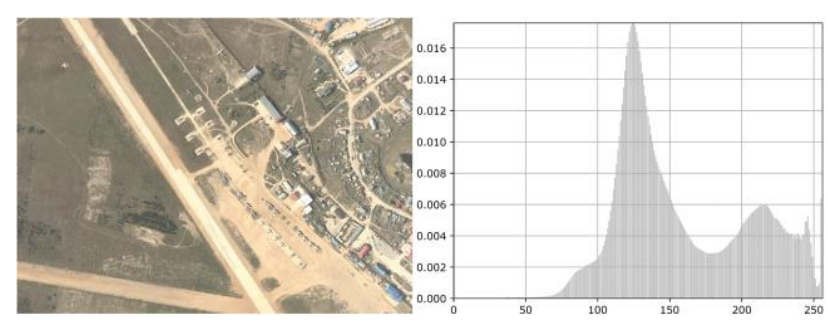

Figure 10. An image with lack of radiometric range in illumination and it's histogram

\subsubsection{Random image noise}

Why: lack of illumination for the surface to be surveyed; increasing the exposure time; increasing the light sensitivity of matrix.

Appearance: randomly placed pixels that differ significantly in brightness or color from the average brightness or color of neighbouring pixels (Khryashchev, 2011; Lapshenkov, 2012).

A high random noise in the image leads to decrease of its information (the disappearance of details) and interpretation properties, an example is shown in Figure 11. 


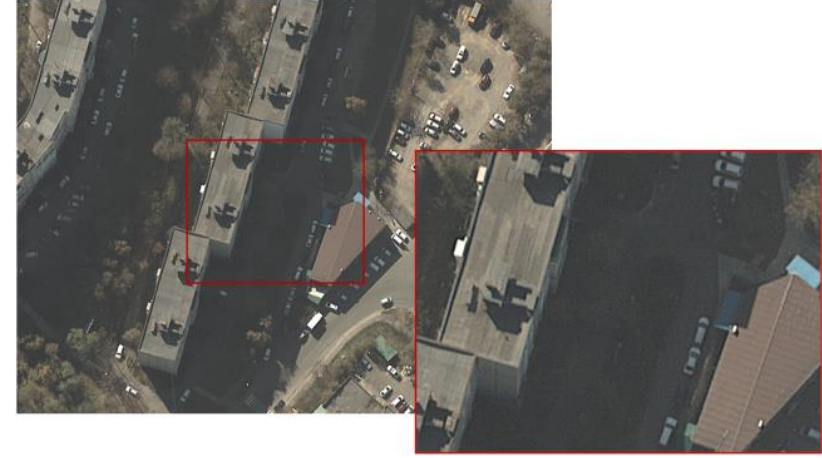

Figure 11. The decrease in information and interpretation quality of an aerial image due to deep shadows and a high random noise

\section{Methods of random image noise assessment:}

visually, at large magnification of flat uniform surfaces (water, flat roofs), areas of shadows from high-rise objects (multi-storey buildings, etc.), as shown in Figure 12, numerically, by calculating the root-mean-square deviation (RMS) of noise, using specialized software that implement the appropriate algorithms (for example, method of harmonic analysis) (Lapshenkov, 2012; Lapshenkov, 2013; Miao, 2019; Chen, Zhang et al., 2019).

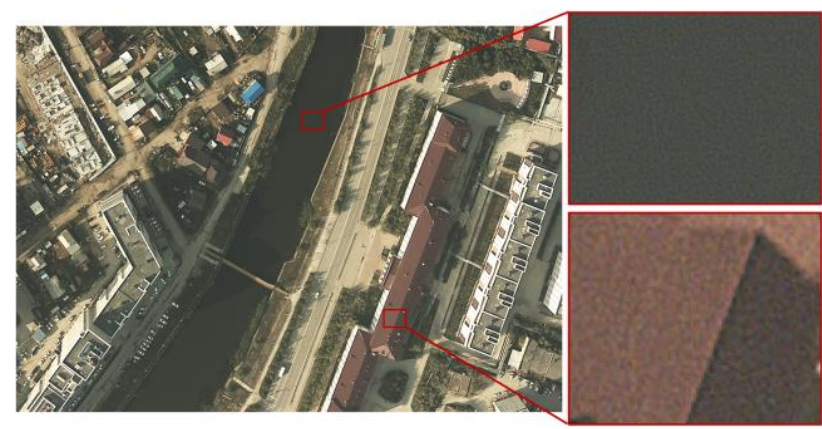

Figure 12. Random image noise, noticeable at high magnification on the water area and on the roof surface

The method of harmonic analysis proposed by Lapshenkov $(2012,2013)$ has proven itself well for assessing the random image noise level. This method allows to determine the random noise RMS (Nrms) in digital image with high accuracy.

Under favourable conditions and correct parameters of aerial surveying, the random noise level for images with radiometric resolution of 8 bits/pixel does not exceed $\mathrm{Nrms}=2,5$ (the number of pixel tones). This value of noise is generalized for wide-format topographic cameras (DMCII, DMCIII, ADS100), for images taken with medium-format topographic cameras (RCD30, PhaseOne), as well as non-metric cameras installed on light UAVs (Sony RX, Canon 5D ProMark) no significant difference for any of the listed camera groups.

Random image noise should not prevent detection of small lowcontrast details in the image, as well as objects in the shadow area.

\subsubsection{Color imbalance}

Why: changing in characteristics of multispectral camera channels, as a result of which they no longer correspond to available calibration parameters; incorrect radiometric profile settings during image post-processing.
Appearance: false colors for individual objects displayed in the image, or predominance of a color tone throughout the image, as shown in Figure 13.

The images should display the underlying surface in natural colors. It should not be individual objects, displayed in image, in false colors or predominance of a color tone throughout the image.

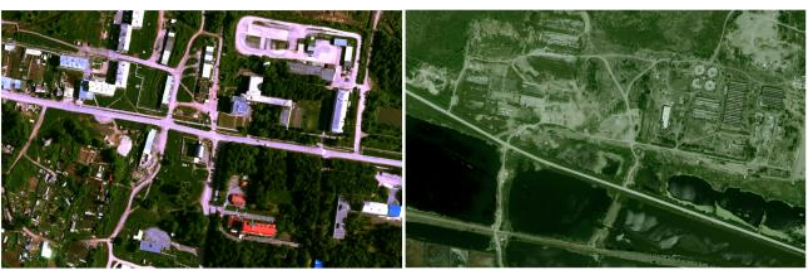

Figure 13. An example of color disbalance in aerial image: false colors for individual objects (left) and the predominance of a color tone throughout the image (right)

\section{Methods of color balance assessment:}

manually by comparing the color components of pixels on gray objects (asphalt pavement, gray roofs, etc.) in any graphics editor (Adobe Photoshop, etc.) that has such ability, the example is shown in Figure 14.

Color components of gray area pixels must be equal, so $R=G=$ $B$. If this equality is not satisfied, an image has a color skew towards the color component, which value is maximum.

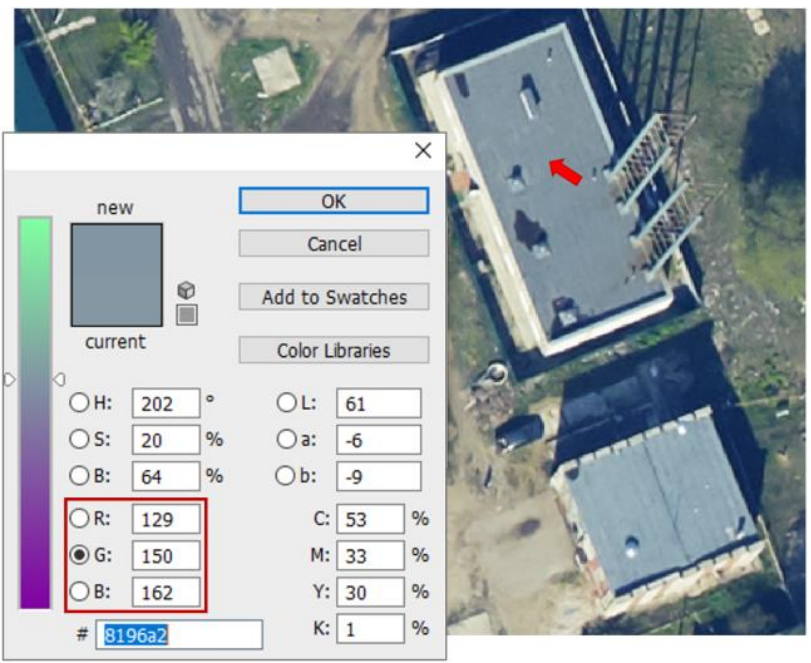

Figure 14. Color balance estimation manually by gray objects in a graphics editor

\section{Methods of color balance assessment:}

software. automatically using specialized algorithms and

The correctness of color rendering (color balance) can be assessed automatically using software that performs the corresponding algorithms (Ancuti et al, 2018; Hussain, Akbari, 2018; Ancuti et al, 2018). One of these algorithms applicable to aerial images is described in Anikeeva (2018).

The acceptable value of color imbalance is determined by visual sense of an image, i.e. the image may be skewed towards any of color components by such number of tone gradations, that is not noticeable by visual examination of an image. Experimentally, it was found that for an image with 8 bits/pixel radiometric resolution, color imbalance becomes noticeable when one of the color components predominates, starting from 10 tones 
compared to the rest, i.e. for gray pixels of an image one of the conditions is met:

$$
\left\{\begin{array}{l}
|R-G| \geq 10 \\
|R-B| \geq 10 \\
|B-G| \geq 10
\end{array}\right.
$$

where $\quad R, G, B=$ red, green, blue color component for gray pixels, respectively

The fulfilment of any inequality from condition (2) corresponds to the value of color imbalance vector $\Delta$, calculated according (3) by the algorithm, proposed in Anikeeva (2018), $\Delta=3$ :

$$
|\vec{\Delta}|=\Delta=\sqrt{\Delta R^{2}+\Delta G^{2}+\Delta B^{2}} \geq 3
$$

where $\Delta R, \Delta G, \Delta B=$ deviation from average (gray) value of red, green, and blue color components for gray pixels, respectively.

Figure 15 shows an example of an image with color imbalance (Figure 15.a) and with correct color balance (Figure 15.b), restored by the color balance correction algorithm (Anikeeva, 2018). The graphs show the color balance breaking for the intervals of pixel values for each of the color components (highlighted in the corresponding color). Figure 15 also shows the values of color imbalance separately for each color component $\Delta \mathrm{R}, \Delta \mathrm{G}$, and $\Delta \mathrm{B}$, as well as the value of the overall color imbalance $\Delta$.

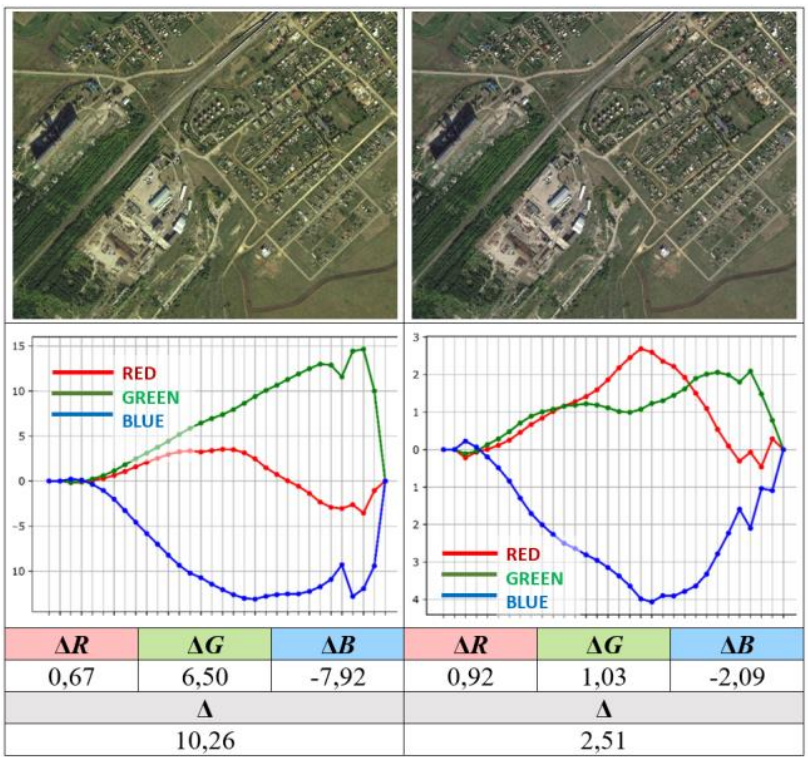

Figure 15.a) Color balance assessment of an aerial image with color imbalance

Figure 15.b) an aerial image with the restored color balance

\subsubsection{Random artifacts}

Why: camera crashes while taking aerial photos; software failure during post-processing of aerial images, other accidental factors (dirty of an airborne camera's hatch glass or the camera lens itself with splashes of water, kerosene, dirt, etc.)

Appearance: corresponding artifacts on the images - spots, stripes, local areas of blurring, etc.

Examples of such imperfections in aerial images, caused by random factors are shown in Figure 16.
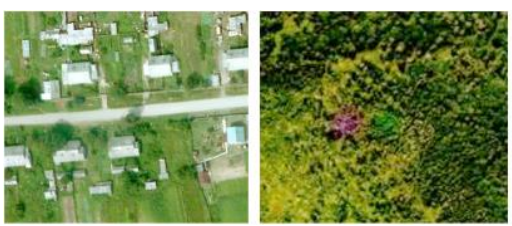

Figure 16.a) Figure 16.b) local areas of artifacts caused by blurring, caused a software failure by water when synthesizing droplets falling a RGB color on the camera image from lens separate spectral

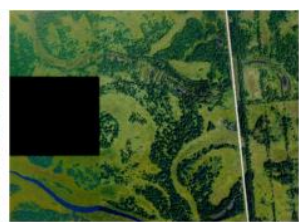

Figure 16.c) artifacts caused by a software failure when creating a single image from composite frames channels

\subsection{Criteria for aerial images quality, obtained for mapping} purposes, and the requirements for them

The analysis of factors that affect the aerial images quality and the resulting imperfections allow to define a set of criteria for them.

Aerial images should have a high quality rates, characterizing with structurometric and gradation (photographic) properties (Kuchko, 1974).

Structurometric properties determine the ability to reproduce small details in the image, these include:

$\square \quad$ sharpness - for images of visually high sharpness, the edge transition's length does not exceed 5 pixels, for numerical assessment the normalized index of image sharpness $l_{\text {norm }}$ should be at least 0,5 ;

spatial resolution - is estimated by MTF using special automated algorithms.

Gradation (photographic) properties allow to estimate the brightness differences reproduction of an object in image. They include:

$\square \quad$ color imbalance - it is visually noticeable in case of one color component predominance starting from 10 tones compared to the rest (for images of 8 bits/pixel radiometric resolution), i.e. the deviation of any one color component for gray pixels must not exceed 10 units of color tone (see condition (2)), or the total color imbalance $\Delta$ must not exceed 3 ;

random image noise - it should not prevent detecting the small low-contrast details in the image as well as objects, located in the shadow areas, where random noise has the greatest impact on the image;

actual radiometric resolution - influenced by various conditions, such as haze, setting of post-processing and others, information completeness - the percentage of information loss in shadows and illumination; imperfections in aerial images caused by influence of random unpredictable factors - the images should not contain artifacts such as spots, stripes, local areas of blurring, etc., caused by crashes of the applied airborne surveying equipment, failures of images post-processing software, or other random factors.

\subsection{Examples of aerial image quality assessment based on proposed criteria system}

The method of image quality assessment based on proposed criteria system can be illustrated by following examples. Figure 17 and 18 show images of different quality, obtained by camera Sony RX, installed on light UAV. In Table 1 and Table 2 the results of image quality assessment are sown. 

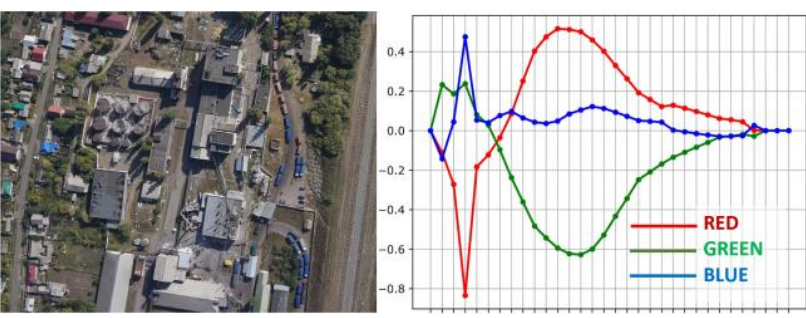

Figure 17. Aerial image, obtained by camera Sony RX, installed on light UAV and graphs of the color balance for each of the color component, the image has good visual quality

\begin{tabular}{|c|c|c|}
\hline \multicolumn{3}{|c|}{ Structurometric properties: } \\
\hline \multirow{2}{*}{ Sharpness } & $\mathrm{X}^{*}$ & 0,53 \\
\hline & $\mathrm{Y}^{*}$ & 0,55 \\
\hline \multirow{2}{*}{ Spatial resolution, pixel } & $\mathrm{X}$ & 1,27 \\
\hline & $\mathrm{Y}$ & 1,27 \\
\hline \multicolumn{3}{|c|}{ Gradation (photographic) properties: } \\
\hline \multirow{4}{*}{$\begin{array}{l}\text { Color imbalance, } \\
\text { number of pixel values }\end{array}$} & in red $\Delta R$ & 0,12 \\
\hline & in green $\Delta G$ & $-0,18$ \\
\hline & in blue $\Delta B$ & 0,05 \\
\hline & total $\Delta$ & 0,22 \\
\hline \multicolumn{2}{|c|}{ Random image noise (RMS), pixel value } & 0,75 \\
\hline \multicolumn{2}{|c|}{$\begin{array}{l}\text { Actual radiometric resolution range, } \\
\text { number of pixel tone values }\end{array}$} & 252 \\
\hline \multicolumn{2}{|l|}{ Haze, pixel value } & 4 \\
\hline \multicolumn{3}{|l|}{ Information completeness: } \\
\hline \multirow{2}{*}{ Loss of information, $\%$} & in shadows & 0,00 \\
\hline & in illumination & 0,00 \\
\hline \multicolumn{2}{|c|}{$\begin{array}{l}\text { Imperfections in aerial images caused by influence } \\
\text { of random unpredictable factors }\end{array}$} & none \\
\hline
\end{tabular}

*calculating direction: $\mathrm{X}$ - along rows, $\mathrm{Y}$ - along columns of an image

Table 1. The quality assessment results of image with good visual quality, shown in Figure 17

The results in Table 1 show, that image quality indicators sharpness and color imbalance do not exceed the recommended values. The RMS of random image noise is less than 1 pixel value, that means that it will not affect the image's interpretation quality. Haze value is not significant, its is not visually noticeable and is detected only by numerical histogram analysis. As it can be seen from Table 1 the image has no losses of information in shadows and illumination, and there is no imperfections, caused by random unpredictable factors. The actual radiometric resolution range is slightly smaller than it's nominal value 256 . due to the haze influence, but this decrease is not significant. Image spatial resolution is close to 1 pixel. Basing on these results, the image's quality can be considered acceptable to provide quality of final geospatial products.

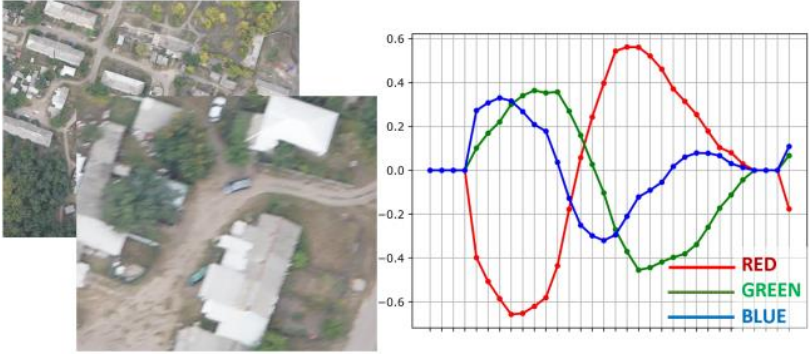

Figure 18. Aerial image, obtained by camera Sony RX, installed on light UAV and graphs of the color balance for each of the color component, the image has poor visual quality

\begin{tabular}{|c|c|c|}
\hline \multicolumn{3}{|c|}{ Structurometric properties: } \\
\hline \multirow{2}{*}{ Sharpness } & $\mathrm{X}$ & 0,48 \\
\hline & Y & 0,29 \\
\hline \multirow{2}{*}{ Spatial resolution, pixel } & $X$ & 1,49 \\
\hline & Y & 1,56 \\
\hline \multicolumn{3}{|c|}{ Gradation (photographic) properties: } \\
\hline \multirow{4}{*}{$\begin{array}{l}\text { Color imbalance, } \\
\text { number of pixel values }\end{array}$} & in red $\Delta R$ & 0,00 \\
\hline & in green $\Delta G$ & $-0,03$ \\
\hline & in blue $\Delta B$ & 0,02 \\
\hline & total $\Delta$ & 0,04 \\
\hline \multicolumn{2}{|c|}{ Random image noise (RMS), pixel value } & 0,78 \\
\hline \multicolumn{2}{|c|}{$\begin{array}{l}\text { Actual radiometric resolution range, } \\
\text { number of pixel tone values }\end{array}$} & 213 \\
\hline \multicolumn{2}{|l|}{ Haze, pixel value } & 43 \\
\hline \multicolumn{3}{|c|}{ Information completeness: } \\
\hline \multirow{2}{*}{ Loss of information, $\%$} & in shadows & 0,00 \\
\hline & in illumination & 0,00 \\
\hline \multicolumn{2}{|c|}{$\begin{array}{l}\text { Imperfections in aerial images caused by influence } \\
\text { of random unpredictable factors }\end{array}$} & none \\
\hline
\end{tabular}

Table 2. The quality assessment results of image with poor visual quality, shown in Figure 18

The results in Table 2 show, that sharpness value is less than recommended 0,5 , it means that there is a blur in the image. The sharpness value on $\mathrm{Y}$-axis (along columns) is significantly lower than on $\mathrm{X}$-axis (along rows), that indicates the motion blur. As a result of blurring, the decrease of spatial resolution to nearly 1,6 pixels. Another factor, that degrades the image quality, is the visually noticeable haze. According to the histogram estimation, its brightness is 43 , that reduces the actual radiometric resolution range to 213 (about $20 \%$ ) in comparison to it's nominal value 256. Results of image quality assessment detected the decline of it's information and interpretation properties, that will not allow to make based on this image geospatial products of an acceptable quality. For this reason, the image (Figure 18) cannot be accepted for further photogrammetric processing.

\section{CONCLUSION}

The problem of quality assessing for aerial imagery, obtained for mapping purposes, now is particularly relevant. The main factors, affecting the visual quality of aerial images, include natural conditions, technical and technological parameters of aerial surveying and the post-processing of the resulting data. Each of these factors individually and their combination in a certain way affect the images quality. 
The images quality assessment should be carried out in terms of structural and gradation (photographic) characteristics. Structural characteristics include sharpness and spatial resolution. Gradation characteristics include color balance, random image noise, radiometric resolution, information completeness - the loss of information in shadows and illumination.

Except the listed indicators, the quality of aerial images may be influenced by random factors, which appearance can't be predicted. Imperfections, caused by these factors, can be detected only by visual examination of an operator. The given quality requirements and criteria are fair and can be applied not only to aerial photo surveying data, but also to satellite imagery, as well as to any cartographic products, providing photorealistic images - orthophotomosaics, 3D terrain models, textured with photographic image, etc.

A unified technique for image quality assessing, based on the proposed criteria system, has a high automation capability because the listed indicators can be calculated automatically. Acceptable values for the listed indicators are the subject of further research.

\section{REFERENCES}

Ancuti, C.O., Ancuti, C., De Vleeschouwer C., Bekaert, P., 2018: Color Balance and Fusion for Underwater Image Enhancement. IEEE Transactions on Image Processing. 27(1), 379-393. doi.org/10.1109/TIP.2017.2759252.

Ancuti, C.O., Ancuti, C., De Vleeschouwer, C., Sbert, M., 2020: Color Channel Compensation (3C): A Fundamental PreProcessing Step for Image Enhancement. IEEE Transactions on Image Processing. 26, 2653-2665. doi.org/10.1109/TIP.2019.2951304.

Anikeeva, I.A., 2018: Method and Algorithm of Automatic Color Rendition Quality Assessment of Digital Aerial and Space Photos. Geodesy and Cartography. 79(7)., 45-56. doi.org/10.22389/0016-7126-2018-937-7-45-56.

Anikeeva, I.A., Kadnichanskiy, S.A., 2017: Evaluation of the Actual Resolution of Digital Aerial and Satellite Imagery Using an Edge Profile Curve. Geodesy and Cartography. 78(6), 25-36. doi.org/10.22389/0016-7126-2017-924-6-25-36.

Chandra, A.M., Gosh, S.K., 2008: Remote Sensing and Geographic Information Systems. Technosphere, Moscow.

Chen, M., Zhang, H., Han, Q., Huang, C.C., 2019: A Convex Nonlocal Total Variation Regularization Algorithm for Multiplicative Noise Removal. EURASIP Journal on Image and Video Processing. doi.org/10.1186/s13640-019-0410-2.

Hussain, M.A., Akbari, A.S., 2018: Color Constancy Adjustment Using Sub-Blocks of the Image. IEEE Access. 6, 46617-46629. doi.org/10.1109/ACCESS.2018.2866792.

Khryashchev, D.A., 2011: About One Method for Determining the Additive Noise Model Most Suitable for the Analyzed Digital Image. Bulletin of the Volgograd State Technical University. 3(76), 24-31.

Kuchko, A.S., 1974: Aerial Photography. Fundamentals and Metrology. Nedra, Moscow.

Lapshenkov, E.M., 2013: Implementation of Methods for Estimating the Image Noise in MATLAB. Bulletin of the Moscow
State University of Instrumentation and Informatics. Instrumentation and Information Technology. 44, 96-106.

Lapshenkov E.M., 2012: Non-Reference Assessment of Digital Image Noise Based on Harmonic Analysis. Computer optics. 36(3), 439-447.

Lim, P.-C., Kim, T., Na, S.-I., Lee, K.-D., Ahn, H.-Y., Hong, J., 2018: Analysis of UAV Image Quality Using Edge Analysis. Int. Arch. Photogramm. Remote Sens. Spatial Inf. Sci., XLII-4, 359364. doi.org/10.5194/isprs-archives-XLII-4-359-2018.

Meißner, H., Cramer, M., and Reulke, R., 2018: Towards Standardized Evaluation of Image Quality for Airborne Camera Systems. Int. Arch. Photogramm. Remote Sens. Spatial Inf. Sci., XLII-1, 295-300, https://doi.org/10.5194/isprs-archives-XLII-1295-2018.

Miao, C., 2019: Research on Denoising Processing of Computer Video Electromagnetic Leakage Reduction Image Based on Fuzzy Degree. EURASIP Journal on Image and Video Processing. doi.org/10.1186/s13640-018-0405-4.

Orych, A., 2015: Review of Methods for Determining the Spatial Resolution of UAV Sensors. Int. Arch. Photogramm. Remote Sens. Spatial Inf. Sci., XL-1/W4, 391-395. https://doi.org/10.5194/isprsarchives-XL-1-W4-391-2015.

Srivastava, G.S., 2014: An Introduction to Geoinformatics. McGraw-Hill Education. New Delhi.

Takahashi,Y., Kuhara, C., Chikatsu, H., 2020: Image Blur Detection Method Based on Gradient Information in Directional Statistics. Int. Arch. Photogramm. Remote Sens. Spatial Inf. Sci., XLIII-B2, 91-95. doi.org/10.5194/isprs-archives-XLIII-B22020-91-2020. 